\title{
Lifestyle, Physical Activity and Cognitive Functions: the Impact on the Scores of Montreal Cognitive Assessment (MoCA)
}

\author{
A. INNOCENTI',2, D.M. CAMMISULI', D. SGROMO', F. FRANZONI ${ }^{3}$, J. FUSI3, \\ F. GALETTA ${ }^{3}$, C. PRUNETI 1,2 \\ ' Department of Clinical and Experimental Medicine, Clinical Psychology Unit and Labs, \\ University of Parma, Parma, Italy; \\ ${ }^{2}$ Centre of Sport Medicine and Physical Exercise Centre (SEM), University of Parma, Parma, Italy; \\ ${ }^{3}$ Department of Clinical and Experimental Medicine, Sport Medicine Unit, University of Pisa, Pisa, Italy.
}

\section{A B S T R A C T}

The Montreal Cognitive Assessment (MoCA) is a rapid screening tool, including subtests assessing some cognitive domains developed to distinguish MCI from normal ageing cognitive changes. Several validation and normative studies around the world considered the influences of age and education on the MoCA score, but none took into account lifestyle and habits. However, lifestyles, and particularly physical activity (PA) have been positively associated with both physical and mental health in elderly. The aim of the present study is to investigate the influence of regularly practiced PA on the MoCA scores in a sample of Italian healthy elderly males. For this purpose, sixty-five healthy adult males, aged 65-81 years were assigned to two groups, physically active group ( $P A G, N=33)$, and sedentary group $(S G, N=32)$, according to recommended levels of physical activity by the World Health Organization. The Italian version of MoCA was administered to each participant. Raw data were analysed by a multiple regression model in which the independent variables were PA, age and education. Results showed a highly significant influence of PA on MoCA total score and on some cognitive domain sub-scores. Furthermore, multiple regression analysis indicated a larger impact on scores by PA than both age and education. In the light of these findings, it is advised to pay attention to the lifestyle of individuals undergoing a neuropsychological evaluation like the MoCa one and, in particular, to the level of PA practiced, as well as age and education.

Key words

MoCA $\bullet$ Aerobic exercise $\bullet$ Cognitive function $\bullet$ Mild cognitive impairment $\bullet$ Neuropsychological test

\section{Introduction}

Mild Cognitive Impairment (MCI) is usually described as a transitional state between age-related cognitive decline and dementia. However, even though it may evolve in Alzheimer's disease or other types of dementia, not all cases of MCI progress to overt dementia (Petersen, 2011; Langa and Levine, 2014; Cheng et al., 2017; Cammisuli and Timpano, 2017). MCI is different from the most severe forms of cognitive decline because it does not fulfil criteria for full-blown dementia, it reports no functional impairment and lack of significant damage in social or occupational functioning (Cammisuli et al., 2012; Cheng et al., 2017; Gallaway et al., 2017; Hughes et al., 2017).

The Montreal Cognitive Assessment (MoCA) is a rapid cognitive screening tool developed to detect MCI from normal ageing cognitive changes taking 10-15 minutes to be administered. It consists of 12 subtasks exploring eight cognitive domains: short-term and delayed verbal memory, 
visuospatial abilities, executive functions, attention, concentration, working memory, language, and orientation to time and place (Nasreddine et al, 2005). MoCA total score ranges from 0 (worst performance) to 30 (best performance), with a cutoff for MCI of 26 in the original version. In MoCa administration it should be taken into account that several items may be culturally biased, in fact several validation studies published all over the world showed great variability in terms of both normative and cut-off scores for detecting people with MCI and AD. Comparisons between healthy people and patients with MCI or AD conducted in several international validations showed wide differences in thresholds, sensitivity and specificity values (Rossetti et al., 2011; Lifshitz et al., 2012; Memória et al., 2013; Conti et al., 2015; Gil et al., 2015; Pirrotta et al., 2015; Santangelo et al., 2015). In particular, for the Italian version of MoCA (Pirani et al., 2006), three independent contributions have been published for MoCA validation (Conti et al., 2015; Pirrotta et al., 2015; Santangelo et al., 2015). These Italian studies found a different cut-off score for healthy subjects of 15 (Pirrotta et al., 2015), 17.5 (Santangelo et al., 2015) and 19.26 (Conti et al., 2015), respectively. The scores are not only lower than those proposed in the original version, but also differ among them. Moreover, both Santangelo et al. (2015) and Conti et al. (2015) showed a significant influence of age and education on MoCA scores, and the population average scores found were different among them, too. Particularly, Santangelo et al. (2015) showed an average total score of $21.98 \pm 4.22$ vs $23.28 \pm 3.22$ found by Conti et al. (2015). Considerably, it seems that demographic characteristics of the participants in the studies could not explain the differences found. In fact, the samples in studies conducted by Pirrotta et al. (2015) and Conti et al. (2015) were comparable, while the sample tested by Santangelo et al. (2015) had an average age lower and an educational level higher than the other studies. Moreover, total score and cut-off score found in Pirotta and Santangelo's studies (2015) are lower than those obtained by Conti et al. (2015).

Even if several of the validation and normative studies all over the world, including the Italian researches, considered the influences of age and education on the MoCA score, we had not found any study taking into account the lifestyle and habits of the subjects tested. However, lifestyles, and particularly physical activity (PA), have been positively associated with both physical and mental health in elderly (Nelson et al., 2007; Biddle et al. 2016; Harridge and Lazarus, 2017; Pruneti et al, 2017).

Strong evidence also showed that PA directly affects the nervous system by interceding on its structural integrity and improving cognitive functioning (Bherer et al., 2013) and seems to have a neuroprotective effect associated to better performance in cognitive tasks in the elderly (Colcombe and Kramer, 2003; Kramer and Erickson, 2007). Furthermore, greater personal activity (leisure or physical one) was consistently associated to a higher level of cognitive ability, starting from the age of 60 (Gow et al, 2012; Pruneti et al., in press), and people who are active in later life present a lower risk of cognitive decline and incident dementia (Berchicci et al., 2015).

Nevertheless, we did not find any study examining the impact of the PA on the MoCa score or other neuropsychological screening tools. In the light of this assumption, the aim of the present study was to investigate the influence of regularly practiced PA on the MoCA raw score in a sample of Italian healthy elderly males.

\section{Methods}

Sixty-five healthy non-smoker adult males, aged 65-81 years (mean 72.1 years $\pm 4.28 \mathrm{SD}$ ) were voluntarily enrolled in the study and assigned to two experimental groups in relation to the amount of practiced PA per week. Subjects who reported to practice walking, jogging or running for 3-4 hours per week, were assigned to the physically active group (PAG, N=33), and those who reported a sedentary lifestyle were assigned to sedentary group ( $\mathrm{SG}, \mathrm{N}=32$ ), according to recommended levels of physical activity by the World Health Organization (2010). Exclusion criteria were the presence of a personal history of cardiac, pulmonary, psychiatric or neurological disorders, diabetes, kidney failure and previously professional sport activities. Each participant gave a written consent to the study and data were anonymously processed by assigning progressive identification codes. Individual and family clinical history, evaluation of PA, lifestyle and education level were assessed using the Eating 
Habits Structured Interview (EHSI) (Pruneti, 2013; Pruneti et al., in press). The EHSI is an interview on lifestyles, medical history and dietary habits planned in 5 sheets and 53 items (master data and medical history, life style, physical activity, body perception, and eating habits). The eating habits sheet included a report of the diet composition of the previous week in order to assess the nutritional pattern of the subjects. The education level of the whole sample was evaluated in years of education. Two senior clinical psychologists individually administered the Italian version of MoCA to each participant in a quiet and comfortable room. Raw scores are considered and no correction for education has been carried out, in order to compare the impact of age, education and PA.

\section{Statistical analysis}

Descriptive statistics (mean, standard deviation, median, and range) for each sample are reported in Table 1. Comparison of demographic characteristics between SG and PAG was performed by Student's $\mathrm{t}$ test, with no statistical significant differences for both age and education levels $(\mathrm{P}>0.5$ and $\mathrm{P}>$ 0.25 respectively). Multiple regression analyses were performed in order to assess the relative influence of PA (assigning 0 to SG and 1 to PAG), age and educational level (expressed as years of schooling) on the subjects' MoCA total score and on each cognitive domain sub-scores. The statistical significance of each multiple regression model was tested by ANOVA, and effect size was calculated by $\operatorname{eta}^{2}\left(\eta^{2}\right)$. Multicollinearity among independent variables was assessed by variance inflation factor (VIF). The statistical adequacy of the multiple linear model was evaluated with the residual analysis applying Shapiro-Wilk and Durbin-Watson tests. When the multiple linear model did not result statistically adequate, we assessed the relative influence of each variable on score by a multiple correlation matrix, applying Spearman's $r_{s}$. Since data included both continuous and ordinal variables, partial correlation coefficients were calculated by Spearman's partial $\mathrm{r}_{\mathrm{s}}$.

The results of the multiple regression analyses were used to formulate a regression equation for esteeming the MoCA total score in function of age, education level and PA. Finally, the average MoCA total score of PAG, SG and the whole sample was compared with the scores shown in the Italian normative studies (Conti et al., 2015; Santangelo et al., 2015), and the effect size was estimated by Cohen's d.

Sub-scores of each domain was computed as described in Santangelo et al. (2015). The investigated domains were the following: memory (score range $0-5$ ), visuospatial skills (score range 0-4), executive functions (score range 0-4), attention, concentration and working memory (score 0-6), language (score 0-6). Spatial and temporal orientation (score 0-6) was not investigated because all subjects totalized the maximum scores obtainable by the test.

\section{Results}

VIF values for PA, age and education were $1.024,1.007$ and 1.020, respectively, showing no collinearity among independent variables. The multiple regression model was found statistically adequate for MoCA total score and for all the domain sub-scores, but attention (Table 2). All scores, but memory sub-score, showed a highly significant influence of PA (Table 3).

Multiple regression analysis showed an influence of all three independent variables on MoCA total score. Standardized coefficient (Beta) and partial correlation coefficient for PA was found to be larger than for age and education, indicating a higher effect of PA than the other ones (Table 3). Executive functions subscore showed significant regression and correlation coefficients for PA but not for age or education (Table 3). Language and visuospatial skills correlation and regression coefficients were statistically significant for both PA and education, but not for age. Beta coefficient and partial correlation coefficient results higher for PA than education, indicating a larger effect of PA (Table 3). On the contrary, memory showed highly significant correlation with age, but not with both PA and education (Tab III). Finally, attention was analysed by Spearman's $r_{s}$, because the multiple regression model was not adequate (Table 2), showing a significant correlation with PA, but not with both age and education (Table 3 ).

By using multiple regression analysis results, we formulated the following predictive equation to estimate MoCA total score in Italian population:

- MoCA total score $=27.337-0.095 \times A G E+$ $3.182 x \mathrm{PA}+0.189 \mathrm{xEDUCATION}$. 
Tab. 1. - Descriptive statistics of demographic caracteristics and MoCA scores for each group.

\begin{tabular}{|c|c|c|c|c|c|c|c|c|c|c|c|c|}
\hline & \multicolumn{4}{|c|}{ PAG N=33 } & \multicolumn{4}{|c|}{ SG N=32 } & \multicolumn{4}{|c|}{ Whole sample $\mathrm{N}=65$} \\
\hline & Mean & SD & Median & Range & Mean & SD & Median & Range & Mean & SD & Median & Range \\
\hline Age & 71.73 & 4.05 & 72 & $65-80$ & 72.41 & 4.55 & 72 & $65-81$ & 72.06 & 4.28 & 72 & $65-81$ \\
\hline Educatin & 8.79 & 3.07 & 8 & $5-13$ & 8.0 & 2.78 & 8 & $5-13$ & 8.40 & 2.94 & 8 & $5-13$ \\
\hline $\begin{array}{c}\text { MoCA } \\
\text { Total Score }\end{array}$ & 25.36 & 1.29 & 26 & $23-27$ & 21.97 & 1.96 & 21 & $19-26$ & 23.69 & 2.37 & 24 & $19-27$ \\
\hline $\begin{array}{l}\text { Executive } \\
\text { Functions }\end{array}$ & 3.73 & 0.52 & 4 & $2-4$ & 2.19 & 0.82 & 2 & $1-4$ & 2.97 & 1.03 & 3 & $1-4$ \\
\hline $\begin{array}{l}\text { Visuospatial } \\
\text { Skills }\end{array}$ & 3.39 & 0.61 & 3 & $2-4$ & 2.56 & 0.91 & 3 & $1-4$ & 2.98 & 0.87 & 3 & $1-4$ \\
\hline Attention & 5.64 & 0.49 & 6 & $5-6$ & 4.84 & 0.63 & 5 & $4-6$ & 5.25 & 0.69 & 5 & $4-6$ \\
\hline Language & 5.45 & 0.56 & 5 & $4-6$ & 4.53 & 0.62 & 4 & $4-6$ & 5.00 & 0.75 & 5 & $4-6$ \\
\hline Memory & 2.03 & 0.59 & 2 & $1-3$ & 2.13 & 0.55 & 2 & $1-3$ & 2.08 & 0.57 & 2 & $1-3$ \\
\hline Orientation & 6 & 0 & 6 & $6-6$ & 6 & 0 & 6 & $6-6$ & 6 & 0 & 6 & $6-6$ \\
\hline
\end{tabular}

\begin{tabular}{|c|c|c|c|c|c|c|c|c|c|c|c|}
\hline & \multicolumn{6}{|c|}{ Multiple Regression Analysis } & \multicolumn{5}{|c|}{ Residual Analysis } \\
\hline & \multicolumn{3}{|c|}{ Coefficients } & \multicolumn{3}{|c|}{ ANOVA } & \multicolumn{3}{|c|}{ Shapiro Wilk } & \multicolumn{2}{|c|}{ Durbin Watson } \\
\hline & $\mathrm{R}$ & $\mathrm{R}^{2}$ & $R^{2}$ adj & $\mathrm{F}_{(3-61)}$ & $P$ & $\eta^{2}$ & W & $P$ & Norm & D & $P$ \\
\hline $\begin{array}{c}\text { MoCA } \\
\text { Total Score }\end{array}$ & 0.78 & 0.61 & 0.59 & 31.23 & $<0.0000$ & 0.61 & 0.982 & $>0.25$ & Y & 2.14 & NS \\
\hline $\begin{array}{l}\text { Executive } \\
\text { Functions }\end{array}$ & 0.77 & 0.59 & 0.57 & 29.43 & $<0.0000$ & 0.59 & 0.978 & $>0.25$ & Y & 1.99 & NS \\
\hline $\begin{array}{l}\text { Visuospatial } \\
\text { Skills }\end{array}$ & 0.57 & 0.33 & 0.29 & 9.83 & $<0.0001$ & 0.33 & 0.987 & $>0.5$ & Y & 2.24 & NS \\
\hline Attention & 0.58 & 0.34 & 0.31 & 10.53 & $<0.0001$ & 0.34 & 0.862 & $<0.001$ & $\mathrm{~N}$ & 2.62 & $<0.05$ \\
\hline Language & 0.70 & 0.49 & 0.47 & 19.68 & $<0.0000$ & 0.49 & 0.970 & $>0.1$ & $Y$ & 2.00 & NS \\
\hline Memory & 0.43 & 0.19 & 0.15 & 4.74 & $<0.005$ & 0.19 & 0.979 & $>0.25$ & $Y$ & 2.04 & NS \\
\hline
\end{tabular}

Note: Independent variables were physical activity ( $S G=0 ; P A G=1$ ), age (in years) and education (in years of schooling). $R$, multiple regression coefficient; adj, adjusted; Norm, normality; NS, not significant; $\eta^{2}$, effect size of ANOVA.

where, 27.337 is the intercept of the multiple regression analysis (Table 3), PA was expressed as binary variable assigning 0 for SG and 1 for PAG, age was expressed in years, and education was expressed in years of schooling. The predictive equation esteemed a score about three points (3.182 $\pm 0.383 \mathrm{SE}$ ) higher in physically active subjects than in sedentary ones.

Finally, MoCA total score of PAG results significantly higher than normative data showed by both Santangelo et al. and Conti et al., whereas SG MoCA score were significantly lower than normative data for Italian population reported by Conti et al, but overlapped the findings of Santangelo et al. (Table 4). Moreover, our whole sample mean MoCA total score overlapped normative data showed by Conti et al., and was significantly higher than that reported by Santangelo et al. (Table 4).

\section{Discussion}

To our knowledge, the present results showed for the first time, the influence of PA on a neuropsychological screening tool such as MoCA. Particularly, we showed that the Italian elderly males practicing a regular aerobic physical exercise totalised a higher MoCA score than sedentary ones. The fact that the standardized coefficient of multiple regression analysis (Beta) was higher for PA than for age or education level clearly 


\begin{tabular}{|c|c|c|c|c|c|c|c|}
\hline \multicolumn{5}{|c|}{$\begin{array}{l}\text { Tab. 3. - Intercepts, multiple regression coefficients and } \\
\text { education for the total MoCA score and for the domain s } \\
\text { Multiple Regression }\end{array}$} & \multicolumn{3}{|c|}{ Partial Correlation Coefficients } \\
\hline & $\mathrm{b}$ & Student- $_{(63)}$ & $\mathrm{P}$ & Beta & Partial R* & $F_{(3-61)}$ & $\mathrm{P}$ \\
\hline \multicolumn{8}{|c|}{ MoCA Total Score } \\
\hline Intercept & 27.337 & 8.27 & $<0.0000$ & & & & \\
\hline Age & -0.095 & 2.13 & $<0.05$ & -0.172 & -0.234 & 3.563 & $<0.05$ \\
\hline$P A$ & 3.182 & 8.31 & $<0.0000$ & 0.676 & 0.722 & 66.536 & $<0.0000$ \\
\hline Education & 0.189 & 2.88 & $<0.01$ & 0.234 & 0.339 & 7.925 & $<0.0005$ \\
\hline \multicolumn{8}{|c|}{ Executive Functions } \\
\hline Intercept & 2.420 & 1.65 & $>0.05$ & & & & \\
\hline Age & -0.009 & 0.46 & $>0.5$ & -0.038 & -0.091 & 0.510 & $>0.5$ \\
\hline$P A$ & 1.492 & 8.81 & $<0.0000$ & 0.729 & 0.764 & 85.574 & $<0.0000$ \\
\hline Education & 0.053 & 1.84 & $>0.05$ & 0.152 & 0.226 & 3.290 & $<0.05$ \\
\hline \multicolumn{8}{|c|}{ Visuospatial Skills } \\
\hline Intercept & 4.848 & 3.04 & $<0.005$ & & & & \\
\hline Age & -0.039 & 1.83 & $>0.05$ & -0.193 & -0.174 & 1.91 & $>0.1$ \\
\hline$P A$ & 0.748 & 4.05 & $<0.001$ & 0.431 & 0.454 & 15.789 & $<0.0000$ \\
\hline Education & 0.072 & 2.27 & $<0.05$ & 0.241 & 0.267 & 4.668 & $<0.01$ \\
\hline \multicolumn{8}{|l|}{ Language } \\
\hline Intercept & 4.546 & 3.68 & $<0.001$ & & & & \\
\hline Age & 0.015 & 0.92 & $>0.25$ & 0.084 & 0.110 & 0.750 & $>0.5$ \\
\hline$P A$ & 0.868 & 6.32 & $<0.0000$ & 0.583 & 0.630 & 40.121 & $<0.0000$ \\
\hline Education & 0.082 & 3.49 & $<0.001$ & 0.322 & 0.413 & 12.526 & $<0.0000$ \\
\hline \multicolumn{8}{|l|}{ Memory } \\
\hline Intercept & 2.809 & 2.37 & $<0.05$ & & & & \\
\hline Age & -0.054 & 3.51 & $<0.001$ & -0.406 & -0.409 & 12.252 & $<0.0000$ \\
\hline$P A$ & -0.150 & 1.14 & $>0.25$ & -0.133 & -0.134 & 1.119 & $>0.25$ \\
\hline Education & 0.024 & 1.06 & $>0.25$ & 0.123 & 0.143 & 1.267 & $>0.25$ \\
\hline \multicolumn{8}{|l|}{ Attention } \\
\hline Age & \multirow{3}{*}{\multicolumn{4}{|c|}{ Multiple regression model was not adequate }} & 0.015 & 0.013 & $>0.95$ \\
\hline$P A$ & & & & & 0.582 & 31.176 & $<0.0000$ \\
\hline Education & & & & & -0.026 & 0.040 & $>0.95$ \\
\hline
\end{tabular}

indicated a stronger influence of PA than of the other two variables. The predictive equation formulated on the basis of the multiple regression results show a difference of 3 points on the MoCA total score between physically active and sedentary Italian elderly males. This confirm the needing to take in account the tested subjects PA levels in both clinical and research when this tool is used.

Furthermore, we found a highly significant impact of PA level on the obtained scores of some cognitive domains investigated by MoCA. Particularly, PA level strongly influences attention, executive functions, language and visuospatial skills, but not memory. We hypothesize that differences found among the several normative and validation studies (Rossetti et al., 2011; Lifshitz et al., 2012; Memória et al., 2013; Conti et al., 2015; Gil et al., 2015; Pirrotta et al., 2015; Santangelo et al., 2015) could be due not only by cultural biases but also by differences in lifestyle, and, particularly, at the 


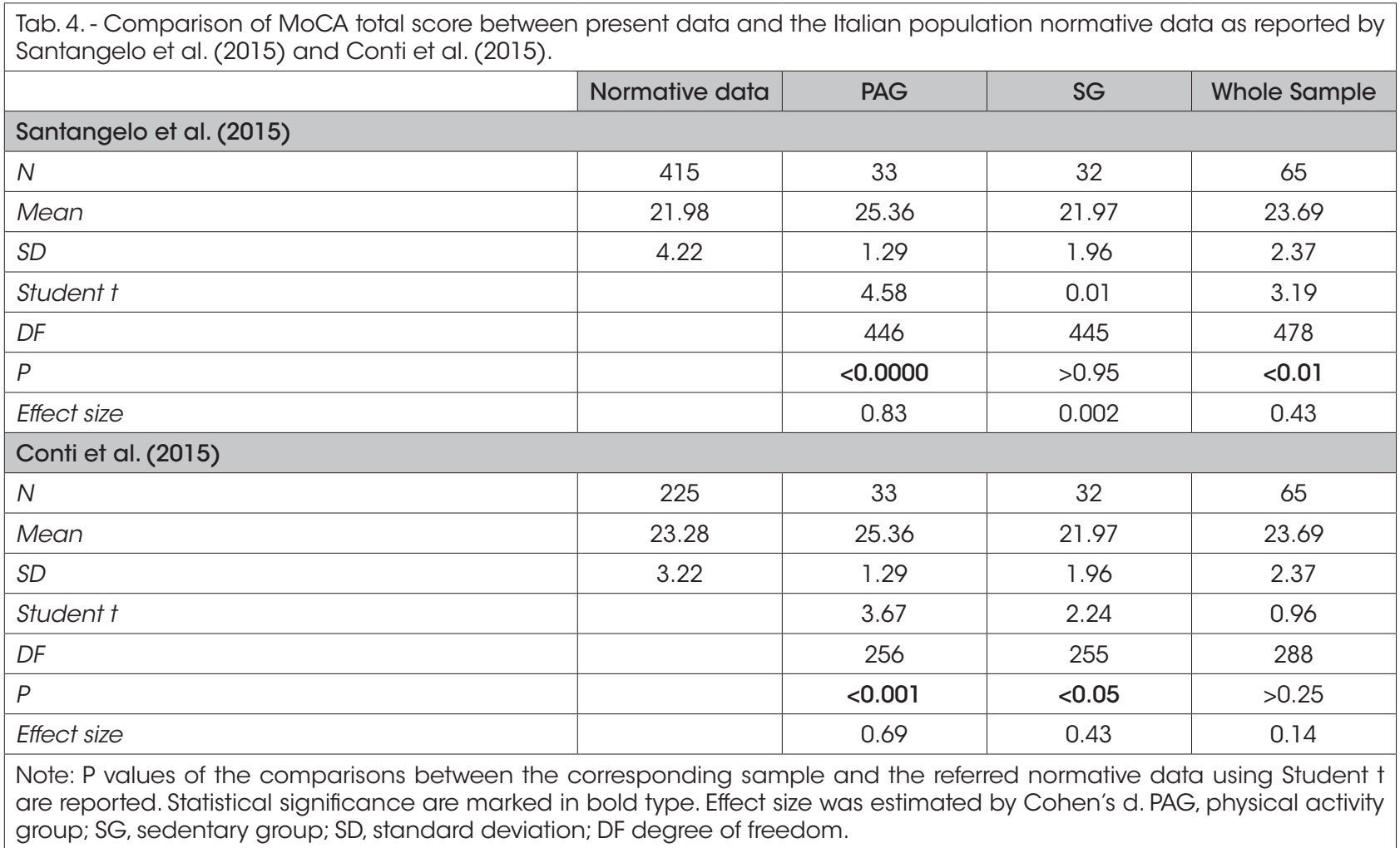

percentage of physically active subjects in the studied samples. Clearly, the differences in PA levels practiced by subjects enrolled in the various studies cannot explain all the discrepancies founded in the MoCA normative data around the world, but they can contribute to reduce the intracultural and intercultural biases. Although our study was conducted only on males, the previous researches on Italian population did not show any gender differences in MoCA scores (Conti et al., 2015; Pirrotta et al., 2015; Santangelo et al., 2015). This could lead to extend our results to females, but this hypothesis should be tested.

On the other hand, present study did not assess the influence on the MoCA scores of cognitive activities like reading, puzzles or playing cards. In light of our findings on PA, we can assume these cognitive activities could similarly affect the MoCA scores. It is well known that both PA and the engagement in cognitive recreational activities more times per week enhance specific cognitive functions in elderly healthy people and may prevent occurring pathological decline over time (Gow et al., 2012; Bherer et al., 2013; Berchicci et al., 2015). Particularly aerobic exercise influence cognitive functioning involving some specific domains, whereas recreational activity with relevant mental involvement (i.e. playing card games) is able to steady improve other specific domains (Pruneti et al., in press). Likewise, Cognitive Reserve (CR) could interact with the various scores. CR theoretical background explains how pre-existing spared cognitive processes may act upon cognitive deficits through compensatory mechanisms, so that an individual with high reserve resists neurological damage longer and shows clinical symptoms only in the advanced phase of the disease (Stern, 2009). It is fascinating to suppose a complementary and, maybe summative, effect of the subjects' PA, cognitive activity and CR in determining the ability of responding to the cognitive tests. Clearly, also these hypotheses will have to be tested and could be material for future researches. In conclusion our findings showed that an assessment of the usual lifestyle and, in particular of PA levels, should be done before the administration of MoCA (and probably this is true for other neuropsychological tests, too), both in clinical and research studies. 


\section{References}

Berchicci M., Lucci G., Perri R.L., Spinelli D., Di Russo F. Benefits of physical exercise on basic visuo-motor functions across age. Front. Aging Neurosci., 6: 48, 2015.

Bherer L., Erickson K.I., Liu-Ambrose T. A review of the effects of physical activity and exercise on cognitive and brain functions in older adults. $J$. Ageing Res., 2013: 657508, 2013.

Biddle S. Physical activity and mental health: evidence is growing. World Psych., 15: 176-177, 2016.

Cammisuli D.M., Timpano Sportiello M. Conscious attention defect and inhibitory control deficit in Parkinson's disease-mild cognitive impairment: A comparison study with amnestic mild cognitive impairment multiple domain. Psychiatr. Danub., in press.

Cammisuli D.M., Timpano Sportiello M., Danti S. Impairment of instrumental extra-memory functions in patients suffering from Mild Cognitive Impairment [Danneggiamento delle funzioni strumentali extra-mnesiche in pazienti affetti da Mild Cognitive Impairment]. G. Gerontol., 60: 255-263, 2012.

Cheng Y.W., Chen T.F., Chiu M.J. From mild cognitive impairment to subjective cognitive decline: conceptual and methodological evolution. Neuropsychiatr. Dis. Treat., 13: 491, 2017.

Colcombe S., Kramer A.F. Fitness effects on the cognitive function of older adults a meta-analytic study. Psychol. Sci., 14: 125-130, 2003.

Conti S., Bonazzi S., Laiacona M., Masina M., Vanelli Coralli M. Montreal Cognitive Assessment (MoCA)Italian version: regression based norms and equivalent scores. Neurol. Sci., 36: 209-14, 2015.

Gallaway P.J., Miyake H., Buchowski M.S., Shimada M., Yoshitake Y., Kim A.S., Hongu N. Physical Activity: A Viable Way to Reduce the Risks of Mild Cognitive Impairment, Alzheimer's Disease, and Vascular Dementia in Older Adults. Brain Sci., 7: 22, 2017.

Gil L., Ruiz de Sánchez C., Gil F., Romero S.J., Pretelt Burgos F. Validation of the Montreal Cognitive Assessment (MoCA) in Spanish as a screening tool for mild cognitive impairment and mild dementia in patients over 65 years old in Bogotá, Colombia. Int. J. Geriatr. Psych., 30: 655-662, 2015.

Gow A.J., Mortensen E.L., Avlund K. Activity participation and cognitive aging from age 50 to 80 in the glostrup 1914 cohort. J. Am. Geriatr. Soc., 60: 1831-1838, 2012.
Harridge S.D., Lazarus N.R. Physical activity, aging, and physiological function. Physiology, 32: 152161, 2017.

Hughes J.C., Ingram T.A., Jarvis A., Denton E., Lampshire Z., Wernham C. Consent for the diagnosis of preclinical dementia states: A review. Maturitas, 98: 30-34, 2017.

Kramer A.F., Erickson K.I. Capitalizing on cortical plasticity: influence of physical activity on cognition and brain function. Trends Cogn. Sci., 11: 342-348, 2007.

Langa, K.M., Levine, D.A. The diagnosis and management of mild cognitive impairment: a clinical review. JAMA, 312b 2551-2561, 2014.

Lifshitz M., Dwolatzky T. Press Y. Validation of the Hebrew version of the MoCA test as a screening instrument for the early detection of mild cognitive impairment in elderly individuals. J. Geriatr. Psych. Neurol., 25: 155-161, 2012.

Memória, C.M., Yassuda, M.S., Nakano, E.Y., Forlenza, O.V. Brief screening for mild cognitive impairment: validation of the Brazilian version of the Montreal cognitive assessment. Int. J. Geriatr. Psych., 28: 34-40, 2013.

Nasreddine Z.S., Phillips N.A., Bédirian V., Charbonneau S., Whitehead V., Collin I., Chertkow H. The Montreal Cognitive Assessment, MoCA: a brief screening tool for mild cognitive impairment. J. Am. Geriatr. Soc., 53: 695-699, 2005.

Nelson M.E., Rejeski W.J., Blair S.N., Duncan P.W., Judge J.O., King A.C., Macera C.A., CastanedaSceppa, C. Physical activity and public health in older adults: recommendation from the American College of Sports Medicine and the American Heart Association. Circulation, 116: 1094-1105, 2007.

Petersen, R.C. Clinical practice. Mild cognitive impairment. New Eng. J. Med., 364: 2227, 2011.

Pirani, A., Tulipani, C., Neri, M. Italian translation of MoCA test and of its instructions. 2016. Retrieved from http://www.mocatest.org/

Pirrotta F., Timpano F., Bonanno L., Nunnari D., Marino S., Bramanti P., Lanzafame P. Italian validation of Montreal cognitive assessment. Eur. J. Psychol. Assess., 31: 131-137, 2015.

Pruneti C., Sgromo D., Merenda J., Cammisuli D.M., Franzoni F., Galetta F., Innocenti A. Physical activity, mental exercise, and cognitive functioning in an Italian sample of healthy elderly males. Neurol. Sci., in press.

Pruneti C. Eating habits structured interview. pp 88-108. In: Pruneti C. Psicopatologia generale. 
L'approccio clinico multidimensionale alla sofferenza psichica con e oltre il DSM. Società Editrice Esculapio, Bologna, 2013.

Rossetti H., Lacritz L., Cullum C., Weiner M. Normative data for the Montreal Cognitive Assessment (MoCA) in a population-based sample. Neurology, 77: 1272-1275, 2011.

Santangelo G., Siciliano M., Pedone R., Vitale C., Falco F., Bisogno R., Siano P., Barone P., Grossi
D., Santangelo F., Trojano, L. Normative data for the Montreal Cognitive Assessment in an Italian population sample. Neurol. Sci., 36: 585-91, 2015.

Stern Y. Cognitive reserve. Neuropsychologia, 47: 2015-2028, 2009.

World Health Organization. Global recommendations on physical activity for health. 2010. Retrieved from http://www.who.int/dietphysicalactivity/ factsheet_recommendations/en/. 